\title{
Spin-flipping polarized electrons
}

\author{
V. S. Morozov, V. A. Anferov,* B. B. Blinov, A. D. Krisch, W. Lorenzon, C. C. Peters, and K. Yonehara \\ Spin Physics Center, University of Michigan, Ann Arbor, Michigan 48109-1120
}

\begin{abstract}
M. Farkhonden, W. A. Franklin, K. D. Jacobs, H. Kolster, S. Sirca, T. Smith, E. Tsentalovich, J. Vieregg, and G. T. Zwart MIT-Bates Linear Accelerator Center, 21 Manning Avenue, Middleton, Massachusetts 01949

E. Six

Arizona State University, Tempe, Arizona 85287

(Received 9 August 2001; published 31 October 2001)

We recently used a prototype rf dipole magnet to study the spin flipping of a $669 \mathrm{MeV}$ horizontally polarized electron beam stored in the presence of a nearly full Siberian snake in the new MIT-Bates storage ring. We flipped the spin by ramping the rf dipole's frequency through an rf-induced depolarizing resonance. After optimizing the frequency ramp parameters, we used multiple spin flipping to measure a spin-flip efficiency of $94.5 \pm 2.5 \%$. The spin-flip efficiency was apparently limited by the field strength in the air-core prototype rf dipole magnet. This unexpectedly high efficiency indicates that very efficient spin flipping of the ring's stored polarized electron beam should be possible using the much stronger ferrite spin flipper, which is now being built by the University of Michigan's Spin Physics Center.
\end{abstract}

DOI: $10.1103 /$ PhysRevSTAB.4.104002

PACS numbers: $29.27 . \mathrm{Bd}, 29.27 . \mathrm{Hj}, 41.75 . \mathrm{Ak}$

Polarized beam experiments are now a major component of the programs in storage rings such as the Indiana University Cyclotron Facility (IUCF) cooler ring [1], the MIT-Bates storage ring [2], the Brookhaven Relativistic Heavy Ion Collider (RHIC) [3], and HERA at DESY [4]. Frequent reversals of the beam polarization direction can significantly reduce the systematic errors in an experiment's spin asymmetry measurements. An rf solenoid was used earlier to spin flip a horizontally polarized proton beam stored in the IUCF cooler ring containing a Siberian snake [5] with $97 \pm 1 \%$ spin-flip efficiency [6,7]. However, the spin rotation due to a solenoid's magnetic field integral decreases linearly with energy because of the Lorentz contraction of its $\int B d l$; thus, a solenoid is impractical for spin flipping in high energy rings. Fortunately, the spin rotation due to a dipole's magnetic field integral is energy independent. Moreover, spin flipping a polarized electron beam may be more complex because, in each turn around a ring, a $1 \mathrm{GeV}$ electron's spin rotation is 1546 times larger than a $1 \mathrm{GeV}$ proton's spin rotation. Therefore, we recently used a prototype rf dipole to spin flip a $669.2 \mathrm{MeV}$ horizontally polarized electron beam stored in the MIT-Bates storage ring with a nearly full Siberian snake.

In any flat circular accelerator or storage ring with no horizontal magnetic fields, each particle's spin precesses around the vertical fields of the ring's dipole magnets. The spin tune $\nu_{s}$, which is the number of spin precessions

\footnotetext{
*Present address: Indiana University Cyclotron Facility, Bloomington, IN 47408-0768.
}

during one turn around the ring, is proportional to the particle's energy

$$
\nu_{s}=G \gamma,
$$

where $\gamma$ is the electron's Lorentz energy factor and its gyromagnetic anomaly, $G \equiv(g-2) / 2=$ $1.15965 \times 10^{-3}$.

This vertical spin precession can be perturbed by the horizontal rf magnetic field from either an rf solenoid or an rf dipole. This perturbation can induce an rf depolarizing resonance, which can be used to flip the spin direction of the ring's stored polarized electrons [6-8]. The frequency $f_{r}$, at which an rf-induced depolarizing resonance occurs, is given by

$$
f_{r}=f_{c}\left(k \pm \nu_{s}\right)
$$

where $f_{c}$ is the electron's circulation frequency and $k$ is an integer. Ramping the rf magnet's frequency through $f_{r}$ can flip the spin. The Froissart-Stora equation [9] relates the beam's polarization after crossing the resonance $P_{f}$ to its initial polarization $P_{i}$,

$$
P_{f}=P_{i}\left\{2 \exp \left[\frac{-\left(\pi \epsilon f_{c}\right)^{2}}{\Delta f / \Delta t}\right]-1\right\},
$$

where $\epsilon$ is the resonance strength, which is determined by the rf magnet's field integral, and $\Delta f / \Delta t$ is the resonance crossing rate, while $\Delta f$ is the frequency range during the ramp time $\Delta t$. In the extreme case of a very strong resonance and a very low crossing rate, the exponential in Eq. (3) becomes very small; then $P_{f}$ is about equal to $-P_{i}$. Thus, the polarization's direction is reversed, while the polarization's absolute value is preserved. 


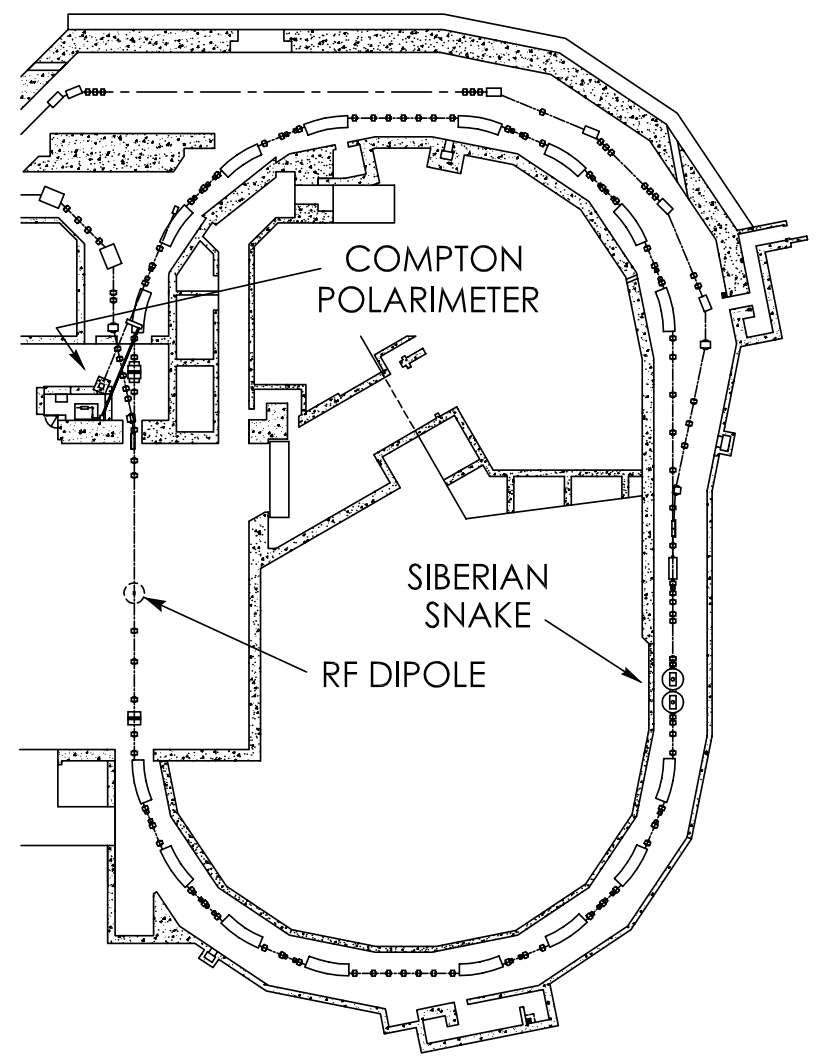

FIG. 1. The MIT-Bates storage ring, showing the rf dipole, the polarimeter, and the Siberian snake.

The apparatus used for this experiment, including the MIT-Bates storage ring [10] and the Compton polarimeter [11], is shown in Fig. 1. We used an rf dipole earlier to study the spin flipping of a $120 \mathrm{MeV}$ horizontally polarized proton beam stored in the presence of a nearly full Siberian snake in the IUCF cooler ring and achieved a maximum spin-flip efficiency of $92.5 \pm 0.5 \%$ [12]. The MIT-Bates rf dipole was a two-turn air-core copper coil with an inductance of about $1 \mu \mathrm{H}$. We used a simple $L C$ resonant circuit to increase the rf dipole's input voltage to $80 \mathrm{~V} \mathrm{rms}$ corresponding to an $\int B d l$ of $0.07 \mathrm{~T} \mathrm{~mm} \mathrm{rms}$. The $669.2 \mathrm{MeV}$ horizontally polarized electron beam in the storage ring was obtained using the MIT-Bates polarized electron source, LINAC, and recirculator [13]. The beam polarization coming out of the source was about $30 \%$. At $669.2 \mathrm{MeV}$, the circulation frequency in the ring was $f_{c}=1.57616 \mathrm{MHz}$.

With a nearly full Siberian snake [14] in the storage ring, the spin tune $\nu_{s}$ was very near, but not exactly equal to, $\frac{1}{2}$. Therefore, at $669.2 \mathrm{MeV}$, Eq. (2) implies that two closely spaced rf depolarizing resonances should be centered around

$$
\frac{1}{2} f_{c}=0.78808 \mathrm{MHz},
$$

with their frequencies at

$$
f_{r}^{-}=f_{c}\left(1-\nu_{s}\right), \quad f_{r}^{+}=f_{c} \nu_{s} .
$$

Since at our energy $G \gamma$ was about 1.518 , the spin tune $\nu_{s}$ varied from 0.518 for no snake to 0.500 for an exactly $100 \%$ snake strength; for example, with a $99 \%$ snake, which rotates the spin by $99 \%$ of $180^{\circ}$, the $f_{r}^{-}$resonance's frequency should be about $0.4997 f_{c}$.

To locate the resonance we first flipped the polarization with the rf dipole using a relatively large frequency ramp range of $16 \mathrm{kHz}$. Then we divided the frequency ramp into two equal parts and continued subdividing the range, always keeping the range containing the spin flip. To reduce systematic errors, each polarization measurement was a matched pair with the beam injected with alternating electron source helicity. These data are shown in Fig. 2 as solid circles; the horizontal bars denote the frequency ranges, while the dashed line shows $\frac{1}{2} f_{c}$. We determined the $f_{r}^{-}$ resonance's frequency to be $0.78775 \pm 0.00025 \mathrm{MHz}$. Note that the maximum spin-flip efficiency was achieved with a frequency range of $\pm 1 \mathrm{kHz}$.

The points shown as open circles all had frequency ranges of $\pm 1 \mathrm{kHz}$, but different central frequencies. Apparently the two frequency ramps, which went well beyond $\frac{1}{2} f_{c}$, crossed both resonances, so that the beam polarization flipped twice with a high spin-flip efficiency; the ramp which did not reach $\frac{1}{2} f_{c}$ did not cross either resonance.

To further study spin flipping, we crossed this $f_{r}^{-}$rfinduced resonance by linearly ramping the rf dipole's frequency from $\frac{1}{2} f_{c}-2 \mathrm{kHz}$ to $\frac{1}{2} f_{c}$ with various ramp times $\Delta t$, while measuring the beam polarization before and after each frequency ramp. The ratio of the radial polarization measured after each ramp to the initial polarization is

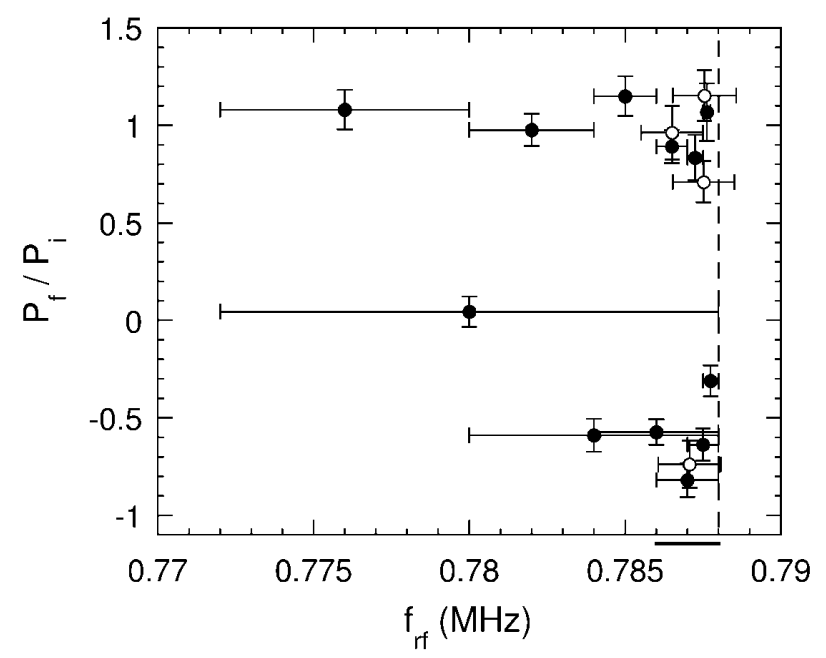

FIG. 2. The ratio of the electron beam's final polarization to its initial polarization at $669.2 \mathrm{MeV}$ is plotted against the center of the frequency ramp. The solid circles show the resonance search data, while the open circles represent the additional data with frequency ranges of $\pm 1 \mathrm{kHz}$. The horizontal bars show the ranges of the frequency ramps; the dashed line shows $\frac{1}{2} f_{c}$. The rf dipole's $\int B d l$ was $0.07 \mathrm{Tmm} \mathrm{rms}$. The thick line at 0.786065 to $0.788070 \mathrm{MHz}$ shows the frequency range $\Delta f$ chosen for use in the later figures. 


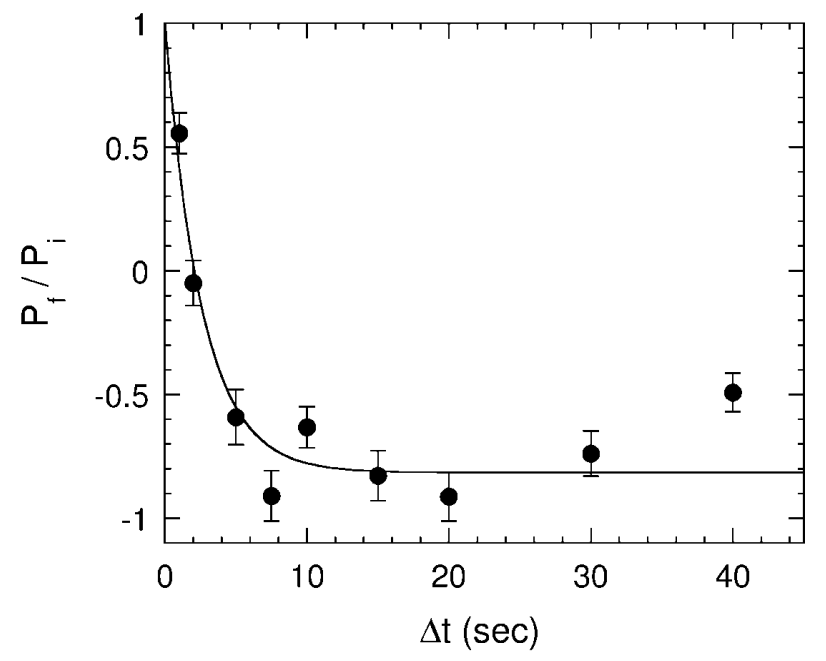

FIG. 3. The ratio of the electron beam's final polarization to its initial polarization at $669.2 \mathrm{MeV}$ is plotted against the $\mathrm{rf}$ dipole's ramp time $\Delta t$. Its frequency range $\Delta f$ was $\pm 1 \mathrm{kHz}$, and its $\int B d l$ was $0.07 \mathrm{~T} \mathrm{~mm} \mathrm{rms}$. The curve is a fit to the data using Eq. (6).

plotted against the ramp time in Fig. 3. The data indicated that the spin-flip efficiency had a broad maximum near $\Delta t=15 \mathrm{~s}$. We ignored the $\Delta t=40 \mathrm{~s}$ point, which was probably partly depolarized by some nearby resonance or some other depolarization mechanism, then we fit all other measured points to a modified [15] Froissart-Stora formula

$$
\frac{P_{f}}{P_{i}}=\left(1+\eta^{\prime}\right) \exp \left[\frac{-\left(\pi \epsilon f_{c}\right)^{2}}{\Delta f / \Delta t}\right]-\eta^{\prime},
$$

where $\eta^{\prime}$ is the spin-flip efficiency due to mechanisms other than the main resonance with strength $\epsilon$. This fit gave a spin-flip efficiency of $\eta^{\prime}=81.6 \pm 4.7 \%$.

After setting $\Delta f$ and the $\mathrm{rf}$ voltage to maximize the spin-flip efficiency, we more precisely determined this efficiency by using a set of multiple spin-flip runs with various ramp times $\Delta t$. For each ramp time $\Delta t$, we flipped the electron beam's polarization three times while measuring its initial polarization and its polarization after each flip. As an example, the multiple-spin-flip data set with $\Delta t=10 \mathrm{~s}$ is shown in Fig. 4, where the ratio of the final radial polarization to the initial radial polarization is plotted against the number of spin flips. The solid circles show data for even numbers of spin flips, while the open circles represent data for odd numbers of spin flips. Notice that, in spite of averaging each data point over both electron source helicities, the data still seemed to have a systematic asymmetry of $25 \pm 7 \%$; this may have been due to some problem in this first use of the new Compton polarimeter [11]. Thus, we fit the data for even and odd numbers of spin flips separately using

$$
\left|\frac{P_{n}}{P_{i}}\right|=\eta^{n}
$$

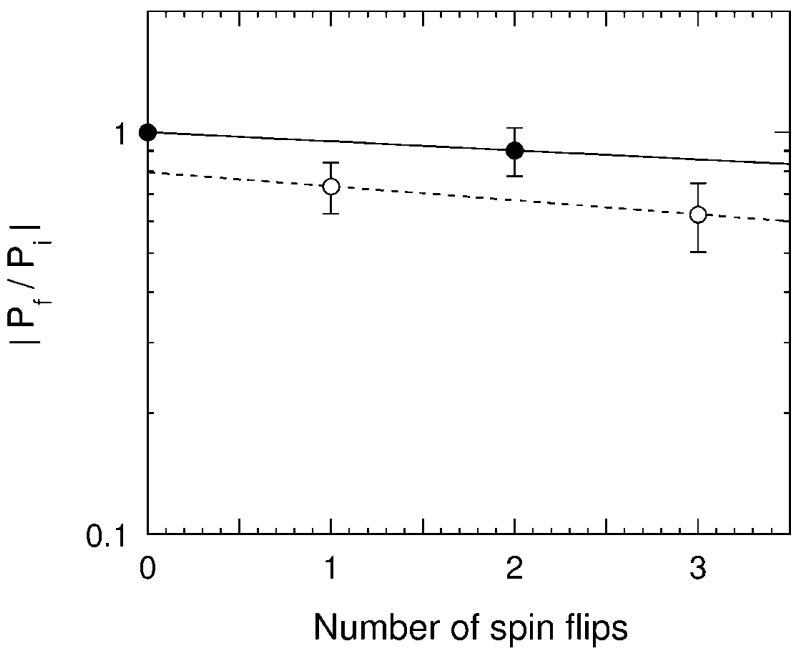

FIG. 4. The ratio of the electron beam's final polarization to its initial polarization at $669.2 \mathrm{MeV}$ is plotted against the number of spin flips. The solid and open circles show the data for even and odd numbers of spin flips, respectively. The rf dipole's frequency ramp time $\Delta t$ was $10 \mathrm{~s}$; its frequency range $\Delta f$ was $\pm 1 \mathrm{kHz}$, and its $\int B d l$ was $0.07 \mathrm{~T} \mathrm{~mm} \mathrm{rms}$. The solid and dashed lines are fits to the data using Eq. (7).

where $P_{n}$ is the measured radial beam polarization after $n$ spin flips, $P_{i}$ is the initial polarization, and $\eta$ is the total single-spin-flip efficiency given by Eq. (6). Note from the essentially equal slopes of the odd-flip and even-flip data that the spin-flip efficiency seems to be independent of this systematic asymmetry. Combining the fitting results for both sets of data we got a spin-flip efficiency of $94.0 \pm$ $4.1 \%$ at this particular $\Delta t$.

Then we plotted the spin-flip efficiency $\eta$ obtained from each multiple spin-flip-versus- $\Delta t$ run in Fig. 5. The dashed line is a hand-drawn curve to guide the eye.

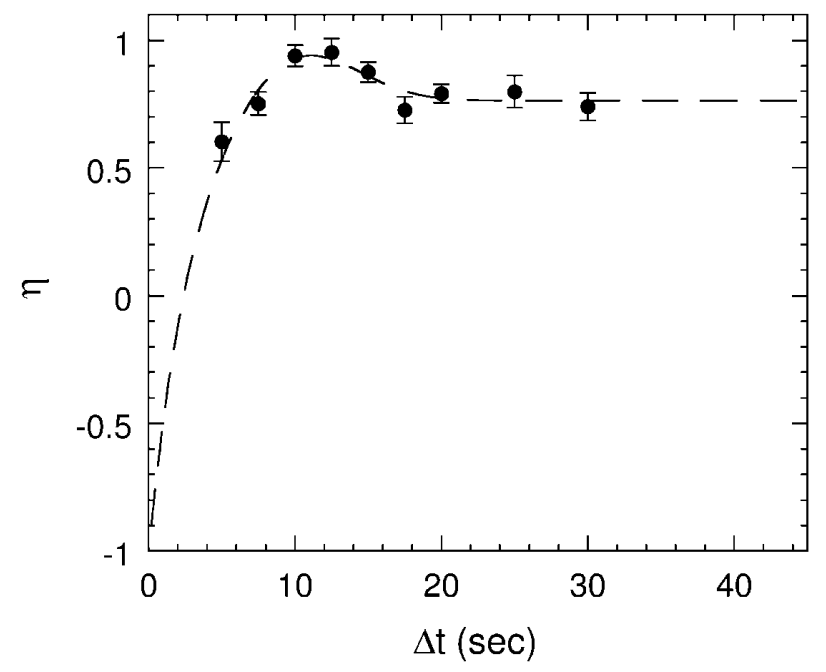

FIG. 5. The efficiency of spin flipping the electron beam at $669.2 \mathrm{MeV}$ is plotted against the rf dipole's ramp time $\Delta t$. Its frequency range $\Delta f$ was $\pm 1 \mathrm{kHz}$, and its $\int B d l$ was $0.07 \mathrm{~T} \mathrm{~mm}$ rms. The dashed line is a hand-drawn curve to guide the eye. 
The spin-flip efficiency reached its maximum at $\Delta t$ of about $12 \mathrm{~s}$ and then dropped for longer ramp times. We observed similar behavior of the spin-flip efficiency earlier in the experiments with a proton beam at IUCF [16]. Averaging of two data points at 10.0 and $12.5 \mathrm{~s}$, where $\eta$ was maximum, gave a measured spin-flip efficiency of $94.5 \pm 2.5 \%$. The spin-flip efficiency was apparently limited by the strength of the rf dipole's field.

In summary, we used a prototype air-core rf dipole to spin flip a stored $669.2 \mathrm{MeV}$ horizontally polarized electron beam with a nearly full Siberian snake in the MIT-Bates storage ring. Using the data from Fig. 5, the maximum measured spin-flip efficiency is $94.5 \pm 2.5 \%$. Thus, this experiment was a successful test of the spin flipping of an electron beam at MIT-Bates. Now we are building a ferrite rf dipole spin-flipper magnet, which will be much stronger than the prototype and have a better magnetic field uniformity due to its ferrite yoke.

\section{ACKNOWLEDGMENTS}

We thank Dr. C. Tschalar, Professor R. Milner, Professor J. Matthews, and the entire MIT-Bates Linear Accelerator Center staff for the successful operation of the new MIT-Bates storage ring with its polarized electron source, LINAC, and recirculator. We are grateful to T. Akdogan, E. Booth, T. Botto, M. Chtangeev, K. A. Dow, and V. Ziskin for their help with developing the Compton polarimeter and the stored polarized beam. We also thank Professor E. D. Courant and Professor A. W. Chao for their help in improving Ref. [15], properly explaining Eq. (6), which seems a useful empirical equation for dealing with more than one isolated depolarizing resonance. This research was supported by two grants from the U.S. Department of Energy.

[1] H. O. Meyer et al., Phys. Rev. Lett. 81, 3096 (1998); B. von Przewoski et al., Phys. Rev. C 58, 1897 (1998).
[2] BLAST Collaboration, R. Alarcon et al., in 1999 Bates Report No. 2-39, 1999.

[3] Y. Makdisi, in High Energy Spin Physics, edited by K. J. Heller and S. L. Smith, AIP Conf. Proc. No. 343 (AIP, New York, 1995), p. 75.

[4] SPIN Collaboration, University of Michigan Reports No. UM-HE 96-20, 1996, and No. UM-HE 99-05, 1999.

[5] Ya. S. Derbenev and A. M. Kondratenko, Part. Accel. 8, 115 (1978).

[6] B. B. Blinov et al., Phys. Rev. Lett. 81, 2906 (1998).

[7] V.A. Anferov et al., in Proceedings of the 13th International Symposium on High Energy Spin Physics (IHEP), Protvino, Russia, 1998, edited by N. E. Tyurin et al. (World Scientific, Singapore, 1999), p. 503.

[8] B. W. Montague, Phys. Rep. 113, 35 (1984).

[9] M. Froissart and R. Stora, Nucl. Instrum. Methods 7, 297 (1960).

[10] M. Farkhondeh et al., in Applications of Accelerators in Research and Industry, edited by J. L. Duggan, AIP Conf. Proc. No. 475 (AIP, New York, 1995), p. 261; K. D. Jacobs, in Intersections of Particle and Nuclear Physics, edited by Z. Parsa and W. J. Marciano, AIP Conf. Proc. No. 549 (AIP, New York, 2000), p. 890.

[11] W. A. Franklin et al., Prog. Part. Nucl. Phys. 44, 61 (2000).

[12] A. M. T. Lin et al., in SPIN 2000: 14th International Spin Physics Symposium, edited by K. Hatanaka and T. Nakano, AIP Conf. Proc. No. 570 (AIP, New York, 2001), p. 736.

[13] K. D. Jacobs et al., in Proceedings of the 1995 Particle Accelerator Conference, Dallas, Texas (IEEE, Piscataway, NJ, 1995), p. 327.

[14] P. M. Ivanov, Yu. M. Shatunov, and G. T. Zwart, MIT-Bates Technical Report No. B/SHR 93-10, 1993.

[15] We know of no formal theoretical derivation of the modified Froissart-Stora formula. It was apparently first developed by some of us in Ref. [6] to better fit its spin-flipping data. We chose an equation similar to the Froissart-Stora formula, but containing a spin-flip efficiency $\eta^{\prime}$ and, in the large resonance strength limit, reducing to our Eq. (7) with $n=1$. The exact form of Eq. (6) was apparently first used in B. B. Blinov et al., Phys. Rev. ST Accel. Beams 3, 104001 (2000). Moreover, when $\eta^{\prime}=1$, Eq. (6) reduces to Eq. (3), which is the normal Froissart-Stora formula.

[16] V. A. Anferov et al., Phys. Rev. ST Accel. Beams 3, 041001 (2000). 